\title{
Velocardiofacial Syndrome Orthodontic \& Surgical Management
}
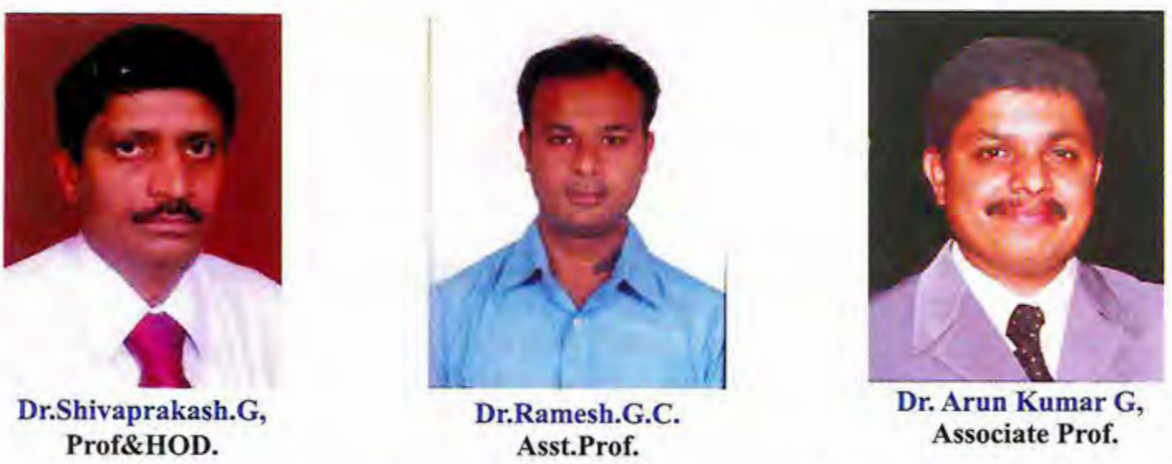

-A case report

\section{Department of Orthodontics \& Dentofacial Orthopaedics, CODS,Davangere.}

Introduction:

Velocardiofacial (VCFS) syndrome comes from the Latin words 'velum' meaning palate, 'cardia' means heart and 'facies' having to do with the face. The most common features are palatal differences $(75 \%)$, heart defects $(75 \%)$, low calcium levels, characteristic facial appearance (vary depending on the individual's ethnic and racial background ), learning problems $(90 \%)$ and $\operatorname{speech}(75 \%)$. This paper presents the combined orthodontic and surgical management of VCFS case

Case report: A 19 year old female patient reported to the Department of orthodontics and Dentofacial Orthopaedics, college of Dental Sciences, Davangere, Karnataka, with chief complaint of irregularly placed upper and lower front teeth. Detailed clinical history revealed that patient had forceps delivery and born with clubfoot, for which the patient was operated at the age of six months.

\section{CLINICALEXAMINATION}
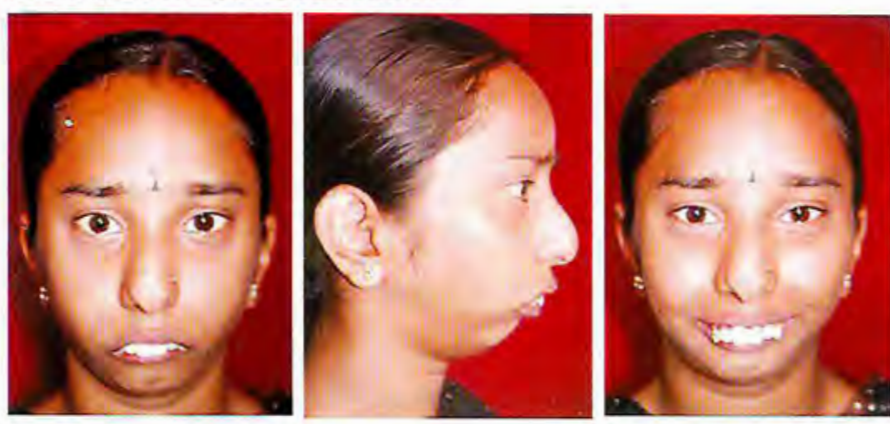

Extra-Oral examination: On frontal examination patient showed, Dolicofacial ,Mesocephalic, Incompetent lips, severly deficient mandible with lack of chinbutton, with cuspid type smile, full crown exposure and $4 \mathrm{~mm}$ gingival exposure. On profile examination, convexity/bulging on the dorsum of the nose, flattened tip of the nose, decreased nasolabial angle and deficient mandible.

Intra-oral examination: Scissor bite irt premolar, canine and Class II molar on right side, complete scissorbite on left side, Overjet of $16 \mathrm{~mm} \&$ closed bite, Retained deciduous canines in the upper arch, palatally placed permanent canines, severe lower anterior crowding \& Missing 36, Square shaped upper and ovoid shaped lower arches.

VELOCARDIOFACIAL syndrome. This patient presented with characteristic feature of syndrome on face, like excess maxilla in both antero-posterior and vertical direction,
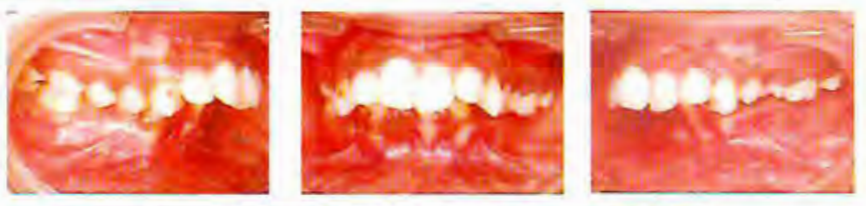

The mandible.On Cephalometric examination, it shows maxillary excess in both antero-posterior and vertical direction, high mandibular plane angle and deficient mandible.On clinical evaluation of the patient, with suspecting syndrome, patient was referred for syndromic evaluation, and the patient was diagnosed as Radiographic Examination: On OPG examination, TMJ appears to be normal with bilaterally symmetrical condyle, missing 36 , erupting $3^{\text {rd }}$ molars in all the quadrants and cant in the mandible.

On Cephalometric examination, it shows maxillary excess in both antero-posterior and vertical direction, high mandibular plane angle and deficient mandible.

On clinical evaluation of the patient, with suspecting syndrome, patient was referred for syndromic evaluation, and the patient was diagnosed as VELOCARDIOFACIAL syndrome. This patient presented with characteristic feature of syndrome on face, like excess maxilla in both antero-posterior and vertical direction, deficient mandible, and flattened nose. But the patient did not presented with any of the cardiac problems. 

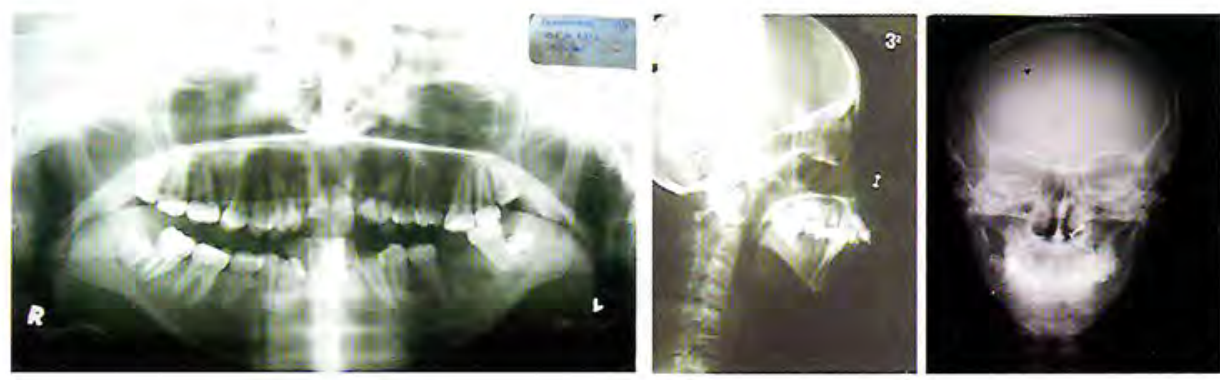

Diagnosis: A female patient named, Annapoorna aged 19 years is diagnosed as case of VELOCARADIOFACIAL SYNDROME characterized by, class II skeletal pattern with maxillary excess and deficient mandible, class II molar \& canine on right \& complete scissor bite on left side, severe crowding in upper \& lower anteriors, retained deciduous maxillary canines, palatally placed maxillary canines, missing 36 , proclined maxillary anteriors, extruded \& severe crowding of lower anteriors, increased midfacial height, decreased lower anterior facial height, increased overjet \& overbite with incompetent lips and convex profile.

Treatment objectives:To improve the facial esthetics,To correct the scissor bite, To relieve lower anterior crowding, To reduce maxillary procumbency, To achieve class I molar and canine relation bilaterally,To achieve ideal overjet and overbite, To correct the mandible and chin button.

Treatment plan: After detailed evaluation of the case, In order to correct both skeletal and dental irregularities, combined Orthodontic and surgical line of treatment was planned. Orthodontic treatment includes $0.022 \mathrm{MBT}$ prescription with extraction of maxillary deciduous canines and all first bicuspids, in which maxillary first bicuspids were planned for extraction on surgical table at the time of surgery. Surgical line included, maxillary impaction, premaxillary setback, differential mandibular advancement along with sliding genioplasty.

\section{Step by step treatment:}
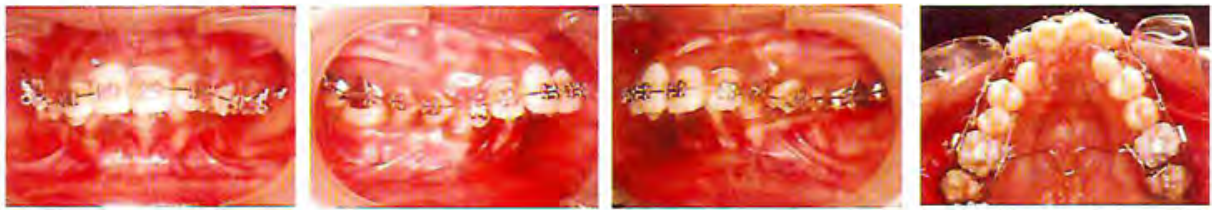

\subsection{Round S S}
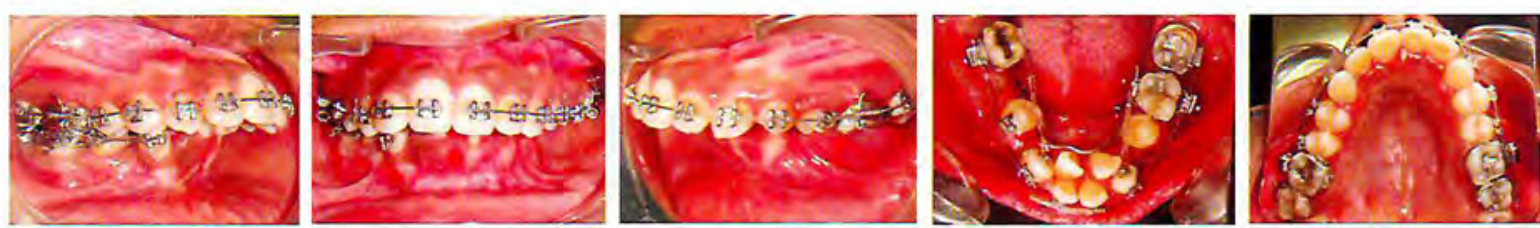

\section{Lower mulligan bypass arch}
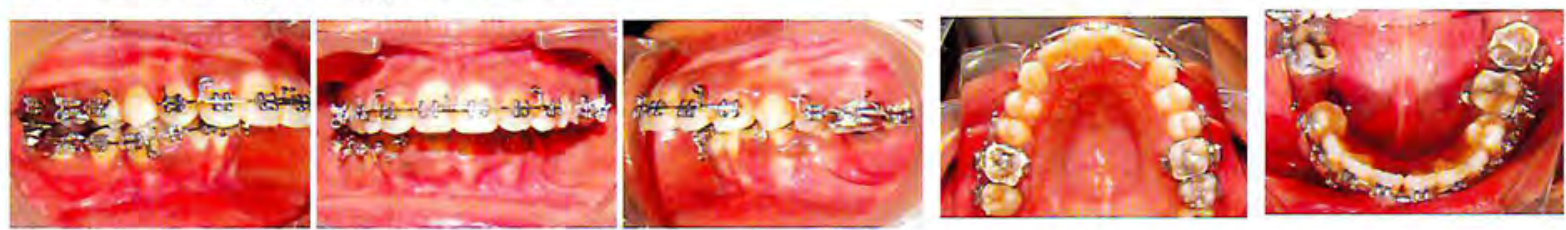

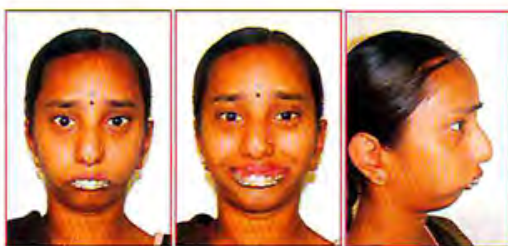

Pre-Surgical Extra Oral.
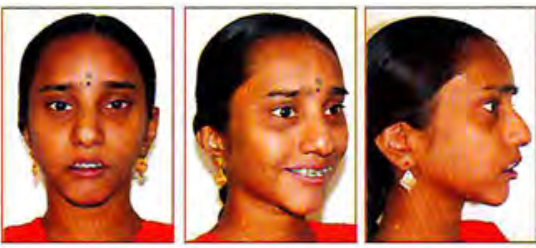

Post-Surgical Extra Oral.
Acknowledgement:

We extend our sincere thanks to: Dr.David P Tauro,Prof \& HOD. Dept. of Oral \& Maxillofacial reconstructive and plastic surgery. CODS, Davangere. 